\title{
New observations of the extended hydrogen exosphere of the extrasolar planet HD 209458b ${ }^{\star}$
}

\author{
D. Ehrenreich ${ }^{1,2}$, A. Lecavelier des Etangs ${ }^{2}$, G. Hébrard ${ }^{2}$, J.-M. Désert ${ }^{2}$, A. Vidal-Madjar ${ }^{2}$, \\ J. C. McConnell ${ }^{3}$, C. D. Parkinson ${ }^{4}$, G. E. Ballester ${ }^{5}$, and R. Ferlet ${ }^{2}$
}

\author{
1 Laboratoire d'astrophysique de l'observatoire de Grenoble, Université Joseph Fourier, CNRS (UMR 5571) BP 53, \\ 38041 Grenoble Cedex 9, France \\ e-mail: david.ehrenreich@obs.ujf-grenoble.fr \\ 2 Institut d'astrophysique de Paris, Université Pierre \& Marie Curie, CNRS (UMR 7095) 98 bis, boulevard Arago 75014 Paris, France \\ 3 Department of Earth and Space Science and Engineering, York University 4700 Keele street, Toronto, ON M3J1P3, Canada \\ 4 Department of Atmospheric, Oceanic, and Space Sciences, University of Michigan 2455 Hayward street, Ann Arbor, MI 48109, \\ USA \\ ${ }^{5}$ Lunar and Planetary Laboratory, University of Arizona 1040 East 4th street, Tucson, AZ 85721-0077, USA
}

Received 25 January 2008 / Accepted 21 March 2008

\section{ABSTRACT}

\begin{abstract}
Context. Atomic hydrogen escaping from the planet HD 209458b provides the largest observational signature ever detected for an extrasolar planet atmosphere. However, the Space Telescope Imaging Spectrograph (STIS) used in previous observational studies is no longer available, whereas additional observations are still needed to better constrain the mechanisms subtending the evaporation process and to determine the evaporation state of other "hot Jupiters".

Aims. Here, we aim to detect the extended hydrogen exosphere of HD 209458b with the Advanced Camera for Surveys (ACS) on board the Hubble Space Telescope (HST) and to find evidence of a hydrogen comet-like tail trailing the planet, whose size would depend on the escape rate and the amount of ionizing radiation emitted by the star. These observations also provide a benchmark for other transiting planets, in the frame of a comparative study of the evaporation state of close-in giant planets.

Methods. Eight HST orbits were used to observe two transits of HD 209458b. Transit light curves were obtained by performing photometry of the unresolved stellar Lyman- $\alpha(\operatorname{Ly} \alpha)$ emission line during both transits. Absorption signatures of exospheric hydrogen during the transit were compared to light curve models predicting a hydrogen tail.

Results. Transit depths of $(9.6 \pm 7.0) \%$ and $(5.3 \pm 10.0) \%$ were measured on the whole Ly $\alpha$ line in visits 1 and 2, respectively. Averaging data from both visits, we find an absorption depth of $(8.0 \pm 5.7) \%$, in good agreement with previous studies.

Conclusions. The extended size of the exosphere confirms that the planet is most likely losing hydrogen to space, yet, the photometric precision achieved does not allow us to better constrain the hydrogen mass-loss rate.
\end{abstract}

Key words. stars: individual: HD 209458 - planets and satellites: general - ultraviolet: stars

\section{Introduction}

Extrasolar planets transiting their parent stars represent $\sim 10 \%$ of the total number of planets detected so far in the solar neighborhood (Schneider 2008). Planetary transits are rare and precious indeed, since they are powerful tools for extracting the key physical properties of planets, like their mean densities, chemical compositions, or atmospheric structures. In fact, during a transit, the stellar light is partially filtered by the planetary atmosphere before reaching the observer, who can thus probe the atmospheric limb structure and composition with a differential spectroscopic analysis.

While detailed models of extrasolar planet transmission spectroscopy have been flourishing (see, e.g., Seager \& Sasselov 2000; Brown 2001; Hubbard et al. 2001; Ehrenreich et al. 2006; Tinetti et al. 2007), few detections of atmospheric signatures have been reported. Most studies have focused on the giant "hotJupiter" HD 209458b, the first extrasolar planet to be observed in transit (Charbonneau et al. 2000; Henry et al. 2000). The presence of atomic species has been proved through the

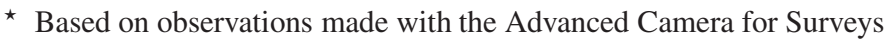
on board the Hubble Space Telescope. supplementary absorption they are triggering at key wavelengths. Sodium (Na I D $\lambda 539 \mathrm{~nm}$ ) in the lower atmosphere of the planet gives rise to a $\sim 10^{-4}$ extra-absorption (Charbonneau et al. 2002). Hot hydrogen ( $\left.\mathrm{HI}^{*}\right)$ in the middle atmosphere provides a $\sim 10^{-4}$ absorption around the Balmer jump (Ballester et al. 2007). Compared to these rather tenuous signals, the $\sim 5 \%$ to $\sim 15 \%$ absorption signatures of hydrogen ( $\mathrm{H} \mathrm{I} \lambda 121.6 \mathrm{~nm}$ ), carbon (C II $\lambda 133.5 \mathrm{~nm}$ ), and oxygen (O I $\lambda 130.2 \mathrm{~nm}$ ) detected by Vidal-Madjar et al. (2003, 2004) seem huge. In fact, those elements are seen in the extended upper atmosphere of the planet, the exosphere.

The proximity of HD $209458 \mathrm{~b}$ to its star ( 0.045 astronomical units) makes the gaseous planet receiving a colossal amount of extreme ultraviolet (EUV) irradiation. Such an energetic input heats the hydrogen upper atmosphere to $\sim 10^{4} \mathrm{~K}$ and inflates it to the Roche limit: the atmosphere is evaporating and its spatial extent produces the large absorption observed in the Lyman- $\alpha$ $(\operatorname{Ly} \alpha)$ stellar emission line (Vidal-Madjar et al. 2003). Elements heavier than hydrogen (C II and O I) are transported upward by the hydrodynamic flow of escaping $\mathrm{H}$ atoms and consequently also give rise to significantly large absorptions (Vidal-Madjar et al. 2004). The evaporation process of the upper atmosphere 
has a solid theoretical ground on which models (Lecavelier des Etangs et al. 2004; Yelle 2004; Tian et al. 2005; García-Muñoz 2007; Lecavelier des Etangs 2007; Penz et al. 2007) are estimating the escape rate $\dot{M}$ of hydrogen and comparing it to observational constraints. So far, theoretical and observational estimations of $\dot{M}$ for HD 209458b are converging (Yelle 2006).

Nonetheless, important questions remain. Is there a hydrogen comet-like tail trailing the planet? Does the size of this cloud depend on the stellar activity and variations in the flux of ionizing EUV radiation? What is the evaporation state of other close-in planets? In fact, observational insights from other systems would significantly constrain and refine the modeling of the evaporation process in a more general frame. However, previous observations were accomplished with the Space Telescope Imaging Spectrograph (STIS) on board the Hubble Space Telescope (HST). The failure of STIS in August 2004 prevented the achievement of additional observations of exoplanets transiting bright stars like HD 209458, HD 189733, or HD 149026.

In addition, Ben-Jaffel (2007) recently challenged the interpretation of Vidal-Madjar et al. (2003) concerning the evaporation of HD 209458b, on the basis of a new analysis of the archival STIS data set. Although the apparent disagreement between those authors has been clarified (Vidal-Madjar et al. 2008), new observations of HD 209458b are required to provide (i) additional observational ground to the evaporation process and (ii) a benchmark observation in the frame of a comparative study of the evaporation state of hot-Jupiters.

These observations were performed with the Advanced Camera for Surveys (ACS; Ford et al. 2003) on board HST; this article describes their analysis.

\section{Observations of HD 209458}

The observing program was originally designed to observe HD 209458 b with HST/STIS. After STIS failure, it has been possible to execute the program with HST/ACS during Cycle 13 . The program (GO\#10145) consists in 3+2 visits, performed with the ACS/High Resolution Camera (HRC) and the ACS/Solar Blind Camera (SBC), respectively. The first 3 visits, aimed at characterizing the near-ultraviolet transmission spectrum of the planet, are under on going analysis (Désert et al., in preparation). We focus here on the last 2 visits, designed for a new observation of the evaporating hydrogen envelope at Ly $\alpha$.

To this purpose, HD 209458b was observed with the HST/ACS/SBC on 2006 May 14 (visit 1) and 2006 May 31 (visit 2). Eight HST orbits in total were used for the following phase coverage of the transit light curve: 3 HST orbits were obtained before 1 st contact, 4 orbits between 1 st and 4th contacts, and 1 orbit after 4th contact. Each HST orbit consists in 8 exposures: a direct image of the star is first made with the F115LP filter; 6 exposures are then acquired in slitless spectroscopy mode with the PR110L prism; another direct image is finally taken with the F115LP filter.

The data reduction described below makes use of the standard calibration (flat-fielded and dark-subtracted) products of the ACS pipeline.

\section{Methods}

Slitless spectroscopy yields spectral images similar to that represented in Fig. 1. The direction of spectral dispersion is given by the spectral trace, which can be linearly approximated for SBC data and make with the detector $x$-axis an angle of $\sim 0.5^{\circ}$.

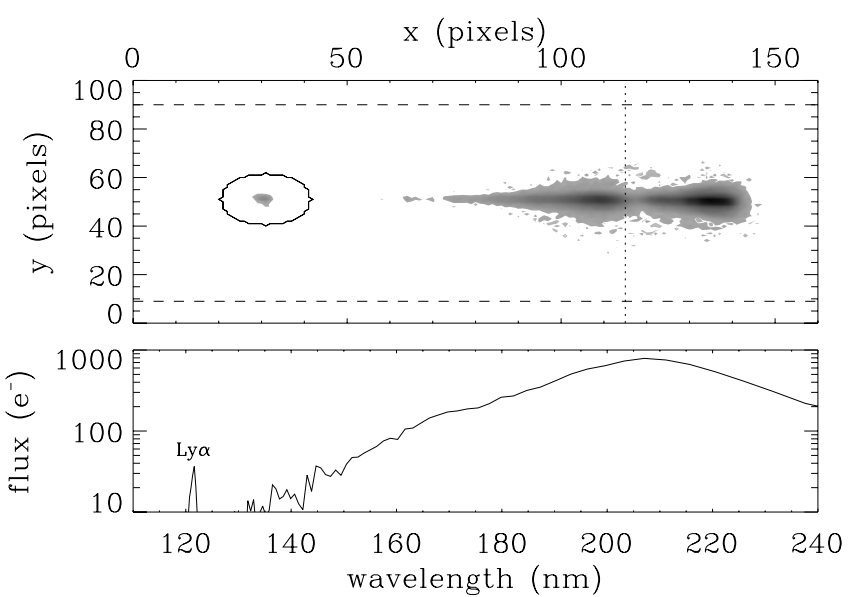

Fig. 1. Median spectrum of HD 209458b. The image in the upper panel is the median of all co-aligned prism exposures. The dispersion direction is close to the detector $x$-axis. The spectrum is unresolved along the $y$-axis. The background is estimated in upper and lower part of the image (above and below the dashed lines). A circular aperture of 10 pixels in radius in represented around the $\operatorname{Ly} \alpha$ emission line. The image is stacked along the $y$-axis to produce the spectrum plotted in the lower panel. The spectrum spans roughly from $\operatorname{Ly} \alpha$ to the dotted line in the image at $240 \mathrm{~nm}$, just after the continuum peak. The bright structure redward to the continuum is an artifact due to the decrease of the resolution, the "red pile-up".

The spectral image has three remarkable structures (from short to long wavelengths): the Ly $\alpha$ stellar emission line, the stellar continuum between 160 and $200 \mathrm{~nm}$, and the "red pile-up". This last feature is an artifact resulting from the built up of photons on a few detector pixels, as the spectral resolution significantly diminishes toward the red. In fact, the spectral resolution of the SBC/PR110L prism is $\lambda / \Delta \lambda \sim 250$ at Ly $\alpha$ (2 pixels at $\left.0.25 \mathrm{~nm} \mathrm{pix}^{-1}\right)$, comparable to the resolution of Vidal-Madjar et al. 's (2004) observations; it is $\sim 40$ ( 2 pixels at $2.5 \mathrm{~nm} \mathrm{pix}^{-1}$ ) at $180 \mathrm{~nm}$, and becomes $\sim 10$ at $330 \mathrm{~nm}\left(2\right.$ pixels at $\left.15 \mathrm{~nm} \mathrm{pix}{ }^{-1}\right)$.

Since the stellar continuum is very low in the ultraviolet typically lower than the background level -, it is convenient to perform aperture photometry on the unresolved $\operatorname{Ly} \alpha$ emission line. In fact, this allows us to make a more direct use of the spectral images provided by the prism, aperture photometry providing the total flux in the Ly $\alpha$ line at a given time. Since the Ly $\alpha$ line is unresolved, no spectral information can be extracted from these data.

The SBC detector is a multi-anode microchannel array (MAMA), i.e., a photon-counting device. Hence, the flux at Ly $\alpha$ is calculated for each image by summing the pixels within a circular aperture of 10 pixels in radius (represented in Fig. 1). We checked that the obtained results do not significantly depend on the size of the photometric aperture for aperture radii chosen between 5 and 20 pixels. Errors on the measured fluxes are calculated from the tabulated error on each pixel in the aperture. Images are previously co-aligned with a reference exposure; the offsets between the spectra in two different images are determined by performing a cross-correlation in Fourier space.

To estimate the background brightness, we used the 2D images (see Fig. 1). On these images, we defined two bands above and below the spectrum along the detector $x$-axis. These bands are 10 pixel-wide in the $y$-direction. For each image at a given position $x$, the background is calculated as the average of the measurements in the bands at the same $x$ position. Since the $x$-axis is close to the dispersion direction, these estimations 


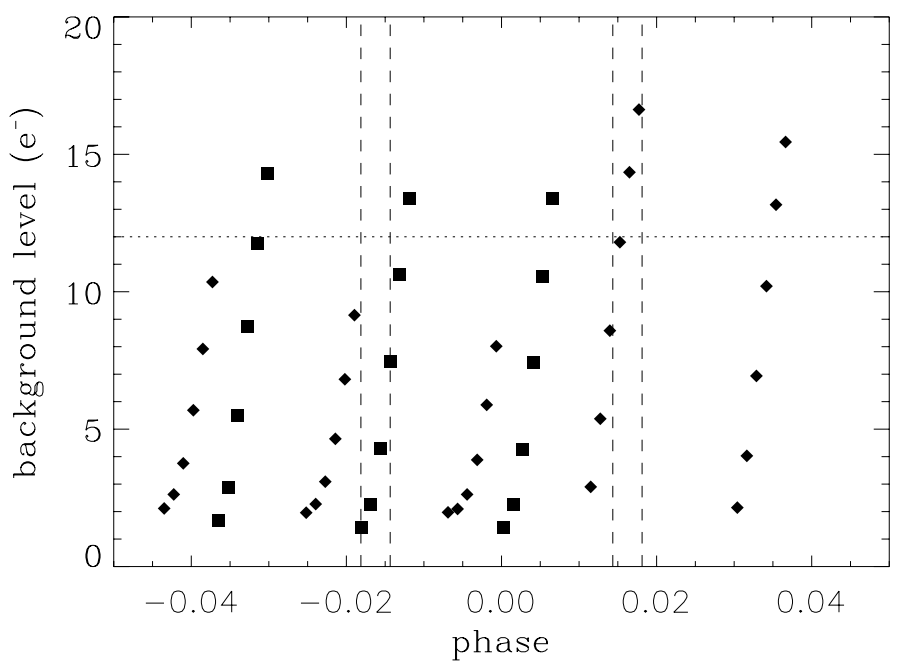

Fig. 2. Background level in individual exposures of visit 1 (filled diamonds) and 2 (filled squares). The median level of the Ly $\alpha$ emission peak is indicated by the dotted line. Dashed lines represent the four contacts of the transit.

should therefore contain any wavelength-dependent structure of the background. The background is fitted along the detector $x$-axis by a 4th-degree polynomial, which is then subtracted to each pixel row of the detector image.

The mean values of the background fits (averaged on the detector $x$-axis) are represented as a function of the orbital phase in Fig. 2. A clear rise of the background is seen along each orbit: it is due to the increase of the geocoronal Ly $\alpha$ line as the spacecraft moves toward the day side of its orbit. This effect is most severe in the last two orbits of visit 1 and all three orbits of visit 2, where the background level during the last exposures reaches the level of $\operatorname{Ly} \alpha$ brightness in the photometric aperture. The peak of the Ly $\alpha$ emission line and is estimated to $\sim 12$ photo electrons $\left(\mathrm{e}^{-}\right)$from the median spectrum of Fig. 1.

Note that the absence of a dispersing slit makes the geocoronal Ly $\alpha$ emission contributing to the flux measured on every pixels of the detector. Therefore, the airglow contamination can be corrected for by performing the background subtraction. These exposures where the signal is overwhelmed by the geocoronal emission (see Fig. 2) are discarded from the photometric time series.

The two time series obtained for visits 1 and 2 are phasefolded, using the ephemeris derived by Wittenmyer et al. (2005), to yield two transit light curves containing 26 and 15 points, respectively. The curves are plotted in upper and middle panels of Fig. 3, where each point represent the weighted mean of all individual exposures within a given HST orbit. We fit to all sub-exposures from each time series a simple trapezoidal transit curve, where the geometry, i.e., the impact parameter and the transit, ingress, and outgress durations, are obtained from accurate photometry of the transit light curve in the optical (Knutson et al. 2007). The transit depth and the out-of-transit baseline level are free to vary to fit the data. Values for these free parameters are then obtained by minimizing a $\chi^{2}$.

\section{Results}

\subsection{The size of the extended exosphere: comparison with previous measurements}

In the first transit (visit 1), we obtain a $\chi^{2}$ of 22.5 for $n=24$ degrees of freedom, or a $\chi^{2} / n \approx 0.94$ and measure a dip of
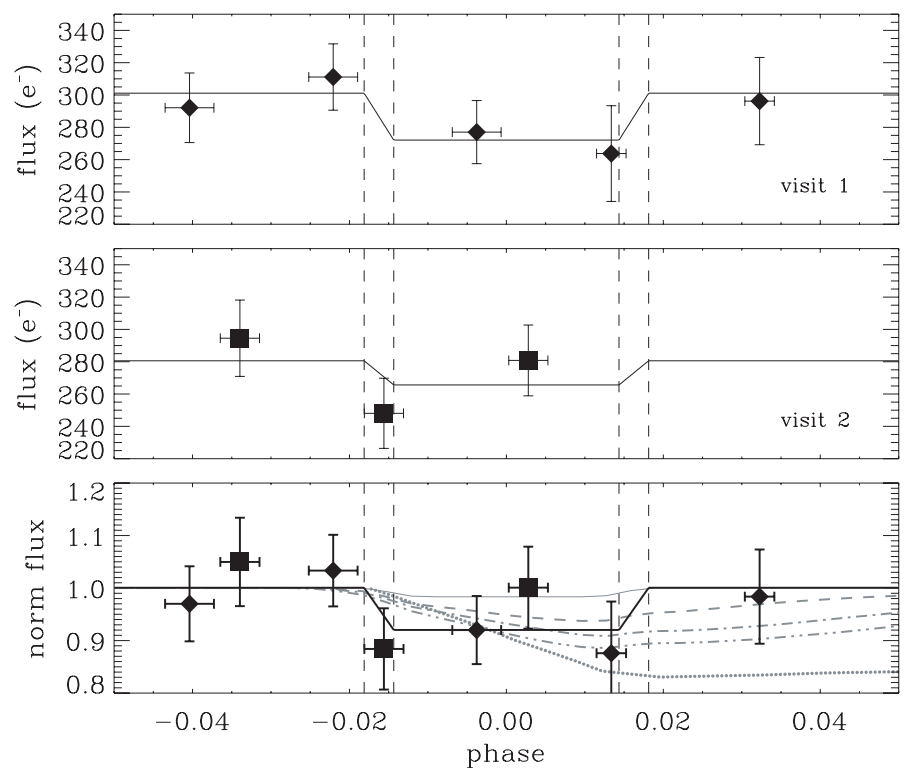

Fig. 3. Light curves obtained during visits 1 (filled diamonds, upper panel) and 2 (filled squares, middle panel). Each data point is the weighted mean of values gathered during one HST orbit. The phase coverage of each $H S T$ orbit is represented by an horizontal error bar. Trapezoidal transit curves are fitted to each time series (plain lines). Contacts are spotted by vertical dashed lines. Each time series is normalized by the out-of-transit flux of its best fit. Resulting values are plotted in lower panel and fitted together with a trapezoidal transit curve (thick black line). The transit depth seen in the visible is indicated for comparison (thin grey line). Predicted light curves of the transit of a hydrogen tail at low spectral resolution produced by two different modeling approaches are also represented in lower panel: (i) assuming the evaporation tail is ionized by the stellar EUV flux (Lecavelier des Etangs, in preparation), for $\dot{M}=1 \times 10^{9} \mathrm{~g} \mathrm{~s}^{-1}$ (dashed line), $\dot{M}=1 \times 10^{10} \mathrm{~g} \mathrm{~s}^{-1}$ (dash-dotted line), and $\dot{M}=3 \times 10^{10} \mathrm{~g} \mathrm{~s}^{-1}$ (dash-dotdotted line); (ii) assuming the evaporation tail interacts with the ionized stellar wind (Schneiter et al. 2007), for $\dot{M}=1 \times 10^{10} \mathrm{~g} \mathrm{~s}^{-1}$ (dotted line).

$(9.6 \pm 7.0) \%$ for an out-of-transit baseline level of $301 \pm 13 \mathrm{e}^{-}$. The fit to the second transit (visit 2) gives a $\chi^{2} / n=16.5 / 13 \approx$ 1.3 , an absorption depth of $(5.3 \pm 10.0) \%$, consistant with visit 1 , and a baseline level of $281 \pm 19 \mathrm{e}^{-}$. Note that the measured errors in the determination of the out-of-transit baseline levels are very close to the theoretical errors expected for a photon-noiselimited signal.

The out-of-transit baseline levels are then used to normalize the respective time series (lower panel of Fig. 3). We finally repeat the above described fitting procedure on normalized time series to obtain a transit depth while considering data points from both visits together. We obtain a depth of $(8.0 \pm 5.7) \%$, in good agreement with individual time series. This last global fit initially yielded a $\chi^{2} / n=67.6 / 40 \approx 1.7$, meaning that the propagated errors underestimate the actual dispersion of the photometry, by a factor of $\sqrt{1.7} \approx 1.3$. The error bars of individual exposures were thus scaled larger by a factor of 1.3 to reflect their true dispersion, and so that the value of $\chi^{2} / n$ for the global fit is close to 1 . This is a conservative approach for the final quoted error bars.

The transit depth in the visible is $\sim 1.6 \%$ (Brown et al. 2001). The observed difference between visible and Ly $\alpha$ measurements is indicative of an additional absorption centered around the $\operatorname{Ly} \alpha$ emission line. The measured absorption is compatible with the $(5 \pm 2) \%$ measured by Vidal-Madjar et al. (2004) 
at an equivalent resolution with $H S T /$ STIS. As discussed in Vidal-Madjar et al. (2008), such a result obtained by measuring the absorption on the whole unresolved Ly $\alpha$ line, is in agreement with other measurements made at a higher resolution. In fact, Vidal-Madjar et al. (2003) measured a transit depth of $(15 \pm 4) \%$ on $\sim 1 / 3$ of the line, while Ben-Jaffel (2007) reported an absorption of $(9 \pm 2) \%$ over $\sim 1 / 2$ of the line.

The absorption of the stellar flux during the transit is approximately equal to the ratio of the planetary to stellar surfaces, $\left(R_{p} / R_{\star}\right)^{2}$. An absorption of $(8.0 \pm 5.7) \%$ thus corresponds to the passage of a spherical hydrogen cloud of radius $R_{\mathrm{H}} \approx$ (0.28 \pm 0.1$) R_{\star}$, where $R_{\star}=1.12 R_{\odot}$ is the stellar radius (Knutson et al. 2007). It gives $R_{\mathrm{H}}=(3.1 \pm 1.1)$ Jovian radii $\left(R_{\mathrm{J}}\right)$, i.e., much larger than the planetary radius of $R_{\mathrm{p}}=(1.32 \pm 0.02) R_{\mathrm{J}}$ measured in the optical by Knutson et al. (2007).

The size of the inflated hydrogen envelope can also be compared to the size of the planet Roche lobe to determine whether the atmosphere is evaporating. The Roche lobe is not spherical but rather elongated in the star-planet direction (Lecavelier des Etangs 2004). In the observational configuration of a transit, the Roche limit of relevance should be taken perpendicular to the star-planet axis; in this case $R_{\text {Roche }} \approx 0.33 R_{\star}$ or $3.7 R_{\mathrm{J}}$ (see Vidal-Madjar et al. 2003, 2008). Considering the size $R_{\mathrm{H}}$ of the hydrogen exosphere and its $1-\sigma$ uncertainty, it is possible that the exosphere reach and exceed the Roche limit.

In fact, we recall that the absorption is measured over the whole Ly $\alpha$ line. Measuring it over the line core, as would be permitted with a higher spectral resolution, would give a larger absorption (Vidal-Madjar et al. 2008), corresponding to a hydrogen envelope extended well beyond the Roche limit. In other words, the velocity of hydrogen atoms responsible for the absorption must largely exceed the planet escape velocity of $\sim 54 \mathrm{~km} \mathrm{~s}^{-1}$ (at the level of the dense atmosphere, far below the exosphere). Vidal-Madjar et al. (2003) reported an absorption in the resolved Ly $\alpha$ line ranging from -130 to $+100 \mathrm{~km} \mathrm{~s}^{-1}$. Their observed $(15 \pm 4) \%$ absorption over this velocity range corresponds to about $(5.7 \pm 1.5) \%$ absorption of the total Ly $\alpha$ line intensity (Vidal-Madjar et al. 2004). The $(8.0 \pm 5.7) \%$ absorption of the total $\operatorname{Ly} \alpha$ line intensity from the present ACS data set could thus even be compatible with hydrogen atoms velocities larger than $\sim 200 \mathrm{~km} \mathrm{~s}^{-1}$.

Because of the observed absorption over the unresolved Ly $\alpha$ line, and from these independent observations only, the hydrogen upper atmosphere must either extend beyond the Roche lobe (if the absorption occurs within the narrow core of the Ly $\alpha$ line), or the atoms velocities must exceed the escape velocity (if the absorption occurs over the whole line or over a broad velocity range of about $\pm 200 \mathrm{~km} \mathrm{~s}^{-1}$ ). Since the atmospheric escape takes place in both cases, the present result is a new independent confirmation of the presence of an extended hydrogen exosphere around HD 209458b, first observed and confirmed by Vidal-Madjar et al. (2003, 2004); it strengthens the atmospheric escape scenario.

\subsection{The hydrogen tail: comparison with models}

The spectral resolution of data analyzed in the present study does not allow us to constrain the velocity of the observed hydrogen and to compare it to the escape velocity, as done by Vidal-Madjar et al. (2003). A clear signature of hydrogen escaping from the planet gravity would be the observation of an escaping hydrogen "cometary-like" tail. Materials expelled from the planet in the observer's direction by effects such as the stellar radiation pressure are seen projected on the plan perpendicular to the line of sight. Because of the almost circular revolution of the planet, the gas tail would hence appear trailing the planet orbit (Rauer et al. 2000; Moutou et al. 2001). Recent predictions of the observational signature of the evaporation tail were provided by Schneiter et al. (2007). They assumed that the planet blows an isotropic neutral hydrogen wind at the escape velocity, with a mass loss rate set as a free parameter. Escaping atomic $\mathrm{H}$ atoms are submitted to gravitational forces from the star and the planet, and interact with the impinging ionized stellar wind. The interaction with the stellar wind, emitted from the star at a rate of $5.7 \times 10^{12} \mathrm{~g} \mathrm{~s}^{-1}$, carves the escaping atmosphere into a comet-like tail (see their Fig. 1). When transiting the star following the planet itself, this tail produces a transit light curve whose shape is changing depending on the assumed mass loss rate $\dot{M}$ of the planet. A simulated light curve with $\dot{M}=1.6 \times 10^{-16} M_{\odot} \mathrm{yr}^{-1}=1 \times 10^{10} \mathrm{~g} \mathrm{~s}^{-1}$ (model M2 in Schneiter et al. 2007), obtained by integrating the flux between -320 and $200 \mathrm{~km} \mathrm{~s}^{-1}$ around the center of the Ly $\alpha$ line, i.e., on the whole line, is plotted over our data points in Fig. 3.

A similar evaporation tail is obtained in simulations performed within our team and used in previous studies (Vidal-Madjar et al. 2003). These simulations include the effect of gravity, stellar radiation pressure - which shapes the evaporation cloud into a comet-like tail -, and stellar EUV ionizing flux - which limits the lifetime of $\mathrm{H}$ atoms escaping from the planet (Lecavelier des Etangs, in preparation). The simulated absorption in the light curve was computed for $\dot{M}=1 \times 10^{9}$, $1 \times 10^{10}$, and $3 \times 10^{10} \mathrm{~g} \mathrm{~s}^{-1}$ assuming a low spectral resolution, on the whole unresolved line, as this is the case for data treated by Vidal-Madjar et al. (2004) and in the present work. The value of the ionizing stellar EUV flux in these simulations was set to the solar value.

Resulting light curves are plotted in Fig. 3 (lower panel). The simulated absorptions over the unresolved Ly $\alpha$ line fit our measurements correctly; however, the precision achieved in the data set does not allow us to put strong constraints on $\dot{M}$. Indeed, the presence of an evaporation tail is mainly constrained by data from the last HST orbit of visit 1. While models of an evaporation tail ionized by stellar EUV radiation are all compatible with our measurement within $1 \sigma$, the model of Schneiter et al. (2007) seems to overestimate the absorption of the hydrogen tail by $\gtrsim 8 \%$ after 4 th contact. We suggest that this might be caused either by the width of the spectral interval chosen to integrate the Ly $\alpha$ absorption, which is barely covering the whole line, or by the value of the stellar wind mass loss rate assumed by these authors.

In addition, Schneiter et al.'s model do not include photoionization, that would decrease the length of the neutral hydrogen tail. Furthermore, none of the models presented here are considering charge exchanges with stellar wind protons that might occur in the exosphere of the planet. In fact, Holmström et al. (2008) show that this last mechanism could produce $\sim 10 \%$ of the escape rate evaluated considering the stellar radiation pressure and gravity (Lecavelier des Etangs et al. 2004). However, because both the stellar radiation pressure and gravity field cannot be neglected in the present situation, the charge exchange process proposed by Holmström et al. is an additional effect rather than an alternative scenario. Hence, it could only enhance the escape rate above the original value evaluated by Vidal-Madjar et al. (2003).

Nonetheless, looking back to our ACS data, more exposures obtained after 4th contact would be needed in order to test the presence of the hydrogen tail, better constrain $\dot{M}$ for a given 
stellar EUV flux, and precise the physical processes contributing to the observed absorption.

\section{Conclusions}

We observed two transits of the extrasolar planet HD 209458b in the stellar Ly $\alpha$ emission line with HST/ACS. Absorption depths of $(9.6 \pm 7.0) \%$ and $(5.3 \pm 10.0) \%$ were measured some 15 -days apart. No significative evidence for a variability in the size of the hydrogen exosphere can be determined from these data.

The extended size of the exosphere is nevertheless confirmed and the absorption obtained by fitting data from both transits, $\Delta F / F=(8.0 \pm 5.7) \%$, is in agreement with previous measurements at similar spectral resolution (Vidal-Madjar et al. 2004). Because of the observed absorption over the unresolved Ly $\alpha$ line, the hydrogen upper atmosphere must extend beyond the Roche lobe, or equivalently the velocity of hydrogen atoms must largely exceed the escape velocity (i.e., the Ly $\alpha$ line is larger than $\sim 200 \mathrm{~km} \mathrm{~s}^{-1}$ ).

This study is limited by the low accuracy of photometry constrained by photon noise. The rise of the sky background during each orbit of the spacecraft, due to the increasing contribution of the geocoronal Ly $\alpha$ emission, is an intrinsic problem of slitless spectroscopy. Higher resolution and more sensitive slit spectroscopy, associated with a better phase coverage of the transit aftermath, are needed to detect deneb-el-Osiris ${ }^{1}$, the hydrogen tail expected by evaporation models.

While studies of the evaporation in other extrasolar planet, like HD 189733b, is still going on with the HST/ACS, high hopes are placed on the repair of STIS and the installation of the Cosmic Origin Spectrograph (COS) during HST Servicing Mission 4.

Acknowledgements. We thank Jeremy Walsh for his help with the scheduling of the ACS observations, Jeffrey Linsky for his promptness to review the manuscript, Sylvestre Lacour for useful discussions, and Michel Mayor for his support. This work is based on observations made with the Advanced Camera for Surveys on board the NASA/ESA Hubble Space Telescope, obtained at the Space Telescope Science Institute, which is operated by the Association of Universities for Research in Astronomy, Inc., under NASA contract NAS 5-26555. These observations are associated with program \#10145. DE acknowledges support of the ANR "Exoplanet Horizon 2009".

\section{References}

Ballester, G. E., Sing, D. K., \& Herbert, F. 2007, Nature, 445, 511

Ben-Jaffel, L. 2007, ApJ, 671, L61

Brown, T. M. 2001, ApJ, 553, 1006

Brown, T. M., Charbonneau, D., Gilliland, R. L., Noyes, R. W., \& Burrows, A. 2001, ApJ, 552, 699

Charbonneau, D., Brown, T. M., Latham, D., \& Mayor, M. 2000, ApJ, 529, L45 Charbonneau, D., Brown, T. M., Noyes, R. W., \& Gilliland, R. L. 2002, ApJ, 568,377

Ehrenreich, D., Tinetti, G., Lecavelier des Etangs, A., Vidal-Madjar, A., \& Selsis, F. 2006, A\&A, 448, 379

Ford, H. C., Clampin, M., Hartig, G. F., et al. 2003, SPIE, 4854, 81

García-Muñoz, A. 2007, Planet. Space Sci., 55, 1426

Henry, G. W., Marcy, G. W., Butler, R. P., \& Vogt, S. S. 2000, ApJ, 529, L41

Holmström, M., Ekenbäck, A., Selsis, F., et al. 2008, Nature, 451, 970

Hubbard, W. B., Fortney, J. J., Lunine, J. I., et al. 2001, ApJ, 560, 413

Knutson, H. A., Charbonneau, D., Noyes, R. W., Brown, T. M., \& Gilliland, R. L. 2007, ApJ, 655, 564

Lecavelier des Etangs, A. 2007, A\&A, 461, 1185

Lecavelier des Etangs, A., Vidal-Madjar, A., McConnell, J. C., \& Hébrard, G. 2004, A\&A, 418, 1

Moutou, C., Coustenis, A., Schneider, J., et al. 2001, A\&A, 371, 260

Penz, T., Micela, G., \& Lammer, H. 2007, A\&A, accepted [arXiv: 0710. 3534]

Rauer, H., Bockelée-Morvan, D., Coustenis, A., Guillot, T., \& Schneider, J. 2000, A\&A, 355, 573

Schneider, J. 2008, The Extrasolar Planets Encyclopædia http://exoplanet. eu

Schneiter, E. M., Velázquez, P. F., Esquivel, A., Raga, A. C., \& Blanco-Cano, X. 2007, ApJ, 671, L57

Seager, S., \& Sasselov, D. D. 2000, ApJ, 537, 916

Tian, F., Toon, O. B., Pavlov, A. A., \& De Sterck, H. 2005, ApJ, 621, 1049

Tinetti, G., Liang, M.-C., Vidal-Madjar, A., et al. 2007, ApJ, 654, L99

Vidal-Madjar, A., Lecavelier des Etangs, A., Désert, J.-M., et al. 2003, Nature, 422, 143

Vidal-Madjar, A., Désert, J.-M., Lecavelier des Etangs, A., et al. 2004, ApJ, 604, L69

Vidal-Madjar, A., Lecavelier des Etangs, A., Désert, J.-M., et al. 2008, ApJ, 676, L57

Wittenmyer, R. A., Welsh, W. F., Orosz, J. A., et al. 2005, A\&A, 632, 1157

Yelle, R. V. 2004, Icarus, 170, 167

Yelle, R. V. 2006, Icarus, 183, 508

1 The tail (deneb in Arab) of the planet Osiris, the nickname of HD 209458b. 